\title{
LA-UR-18-24026
}

Approved for public release; distribution is unlimited.

Title: $\quad$ Report May 2018 - High-Resolution 3D Acoustic Borehole Integrity Monitoring

Author(s): $\quad$ Pantea, Cristian

Intended for: $\quad$ Report

Issued:

2018-05-09 
Disclaimer:

Los Alamos National Laboratory, an affirmative action/equal opportunity employer, is operated by the Los Alamos National Security, LLC for the National Nuclear Security Administration of the U.S. Department of Energy under contract DE-AC52-06NA25396. By approving this article, the publisher recognizes that the U.S. Government retains nonexclusive, royalty-free license to publish or reproduce the published form of this contribution, or to allow others to do so, for U.S. Government purposes. Los Alamos National Laboratory requests that the publisher identify this article as work performed under the auspices of the U.S. Department of Energy. Los Alamos National Laboratory strongly supports academic freedom and a researcher's right to publish; as an institution, however, the Laboratory does not endorse the viewpoint of a publication or guarantee its technical correctness. 


\section{Report May 2018 - High-Resolution 3D Acoustic Borehole Integrity Monitoring}

\section{Quarterly Research Performance Progress Report}

Federal Agency and Organization: Office of Fossil Energy (FE); National Energy Technology Laboratory (NETL); Geologic Storage Technologies

Recipient Organization: Los Alamos National Laboratory

Project Title: High-Resolution 3D Acoustic Borehole Integrity Monitoring

Report Submitted by: $\quad$ Cristian Pantea

Research Scientist 4

pantea@lanl.gov

505-665-7598

Date of Report Submission: May 7, 2018

Reporting Period: Oct 1, 2017 to Mar 31, 2018

Project Partners:

- LANL: Cristian Pantea (PI), Dipen Sinha, Eric Davis, Vamshi Chillara, Craig Chavez, Jacob Verburg, , Yu Chen, Lianjie Huang

- NETL: $\quad$ Barbara Kutchko, Dustin Crandall

- WVU: $\quad$ Roger Chen

- SNL: $\quad$ Douglas A. Blankenship, Jiann-Cherng Su

- ORNL: Hector Santos-Villalobos

- no cost-sharing partners

\section{DOE Project Team:}

DOE Project Officer - Mark Ackiewicz
Traci Rodosta
Project Monitor - Mary Kylee Rice

All reports should be written for public disclosure. Reports should not contain any proprietary or classified information, other information not subject to release, or any information subject to export control classification. If a report contains such information, notify DOE within the report itself. 


\section{Table of Contents}

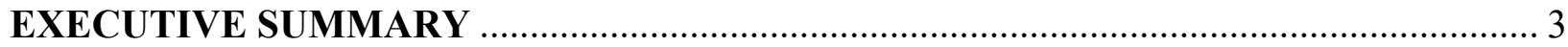

ACCOMPLISHMENTS \& MILESTONE UPDATE ...................................................... 3

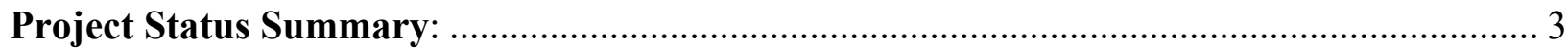

Task 1. Investigation of existing technology ....................................................... 3

Task 2. Define metrics ..................................................................................... 9

Task 3. Industry partners/technology maturation plan ............................................. 10

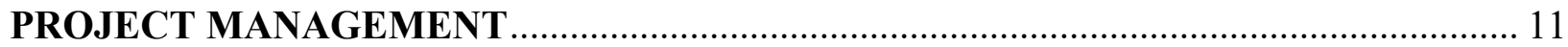

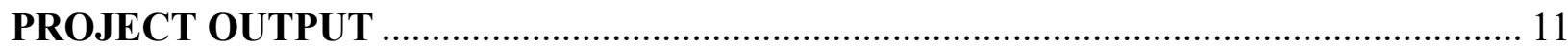

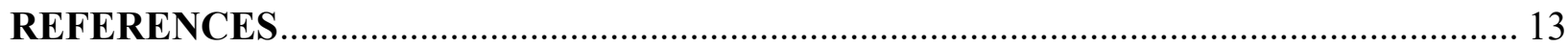

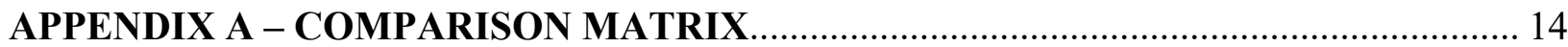

All reports should be written for public disclosure. Reports should not contain any proprietary or classified information, other information not subject to release, or any information subject to export control classification. If a report contains such information, notify DOE within the report itself. 


\section{EXECUTIVE SUMMARY}

SubTER Topic 1. Wellbore Diagnostics and Integrity Assessment

Real-time, in-situ, high spatial resolution (sub-cm) imaging of the near-borehole environment would revolutionize wellbore diagnostics and integrity assessment by direct observation of defects. It is becoming increasingly apparent, that better understanding of the near-wellbore environment is required to meet the safety and operational needs in challenging environments such as those present in subsurface energy extraction (geothermal) and storage (CO2 sequestration) applications. Therefore, it is important to have a more robust ability to image the near-borehole and reliably detect defects.

It was proposed to further develop and improve our advanced $3 \mathrm{D}$ imaging system to evaluate casing defects (e.g. corrosion) and cement quality in either open- or cased-borehole with the ultimate goal to develop a commercially deployable technology. The system consists of a unique acoustic source (LANL) and advanced inversion techniques for image processing (LANL, ORNL). This system will provide comprehensive borehole integrity monitoring with improved resolution over existing techniques. As an application of this imaging system, we will characterize the effectiveness of next-generation wellbore completion technology (NETL, SNL), and will demonstrate that, unlike current technology, the proposed approach can successfully characterize foamed cements.

The first 6 months of the project were dedicated to Phase 1: Feasibility study. Assess feasibility of the high-resolution 3D imaging technique for wellbore integrity applications, across multiple industries (geothermal, CO2 sequestration, oil \& gas, nuclear repository).

\section{ACCOMPLISHMENTS \& MILESTONE UPDATE}

\section{Project Status Summary:}

\begin{tabular}{|c|l|c|c|}
\hline Task & \multicolumn{1}{|c|}{ Title } & $\begin{array}{c}\text { Planned } \\
\text { Completion } \\
\text { Date }\end{array}$ & $\begin{array}{c}\text { Completion Date } \\
\text { or \% completion }\end{array}$ \\
\hline 1 & Investigation of existing technology & $03 / 31 / 2018$ & $100 \%$ \\
\hline 2 & Define metrics & $03 / 31 / 2018$ & $100 \%$ \\
\hline 3 & Industry partners/technology maturation plan & $03 / 31 / 2018$ & $100 \%$ \\
\hline
\end{tabular}

Task 1. Investigation of existing technology

\section{Planned Activities:}

Baseline performance of existing technologies, validated through elicitation of industry input, case studies, and/or literature review.

All reports should be written for public disclosure. Reports should not contain any proprietary or classified information, other information not subject to release, or any information subject to export control classification. If a report contains such information, notify DOE within the report itself. 


\section{Actual Accomplishments:}

Performed a comprehensive literature/existing technology study for wellbore integrity monitoring tools.

\section{Literature study:}

Wellbore integrity is crucial for geothermal energy production. It is necessary to monitor wellbore integrity throughout the life cycle of production/injection wells. The existing techniques for wellbore integrity monitoring include optical, electrical and acoustic imaging.

Optical imaging uses photographic camera for visual inspection and recording of downhole completion conditions [Prensky, 1999]. It is capable of directly obtaining a high-resolution color image of the wellbore wall for fracture identification or casing inspection. The directimaging technology has only limited use because of the operational requirement of a transparent borehole fluid or in air holes, which are often impractical. The other drawback of optical imaging is that the technique can detect only fractures on the inner surface.

Acoustic imaging makes use of acoustic reflectivity to detect the changes of surface roughness and fractures in the wellbore wall. Acoustic waves travel through the drilling fluid and undergo partial reflections on the borehole wall. Reflected pulses are received by a transducer. The amplitudes and travel times of the reflected pulses are influenced by several factors, such as fractures, vugs, breakouts on the borehole walls, and acoustic impedance between the fluid and the borehole walls [Zemanek et al., 1970; Massiot et al., 2015]. The wellbore televiewer can provide a $360^{\circ}$ image in an open or cased hole. The principle limitation of current state-of-the-art of this technique is that it uses only acoustic reflections, and can detect only the inner surface of the wellbore walls.

Electric imaging identifies fractures by contrasts in conductivity between the fracture and the adjacent borehole wall. The images are produced by placing pads with arrays of electrodes maintained at a constant electrical potential against the borehole wall, and measuring the current drop as the electrodes travel along the borehole wall. Data from multiple electrodes are combined to produce electrical conductivity images [Davatzes and Hickman, 2005]. Electric imaging tools have proved superior to the acoustic imaging in the identification of some structural features and rock properties in depth. However, acoustic imaging can reveals a higher resolution on the wellbore surface [Davatzes and Hickman, 2005].

A more streamlined table is presented in APPENDIX A, in a comparison table. Only three main competitors are presented, with a detailed comparison of different parameters of interest, e.g. frequency, collimation resolution.

Tools available on the market:

\begin{tabular}{|c|c|c|c|c|c|}
\hline Technique & $\begin{array}{l}\text { Compa } \\
\text { ny }\end{array}$ & Range & $\begin{array}{l}\text { Frequenc } \\
\text { y }\end{array}$ & Resolution & Notes \\
\hline $\begin{array}{l}\text { FMI - Fullbore } \\
\text { Formation } \\
\text { Microimager }\end{array}$ & $\begin{array}{l}\text { Schlum } \\
\text { berger }\end{array}$ & 30 inches & $\mathrm{N} / \mathrm{A}$ & $5 \mathrm{~mm}$ both & $\begin{array}{l}\text { Temp } 175 \\
\mathrm{C} / \mathrm{pressure} 20 \mathrm{ksi} / \\
80 \% \text { of borehole in } 8 \\
\text { inch hole coverage }\end{array}$ \\
\hline
\end{tabular}

All reports should be written for public disclosure. Reports should not contain any proprietary or classified information, other information not subject to release, or any information subject to export control classification. If a report contains such information, notify DOE within the report itself. 


\begin{tabular}{|c|c|c|c|c|c|}
\hline $\begin{array}{l}\text { IBC - Imaging } \\
\text { Behind Casing }\end{array}$ & $\begin{array}{l}\text { Schlum } \\
\text { berger }\end{array}$ & $\begin{array}{l}\text { Casing- } \\
\text { formation } \\
\text { interface }\end{array}$ & $\sim 200 \mathrm{kHz}$ & $\begin{array}{l}102 \mathrm{~mm} \text { vert } \\
30 \mathrm{~mm} \text { azim }\end{array}$ & $\begin{array}{l}\text { Good for low- } \\
\text { impedance } \\
\text { cements/ produces } \\
\text { SLG maps / can } \\
\text { possibly see casing } \\
\text { position within } \\
\text { borehole }\end{array}$ \\
\hline $\begin{array}{l}\text { CBL - Cement } \\
\text { Bond Log } \\
\text { (multiple tools) }\end{array}$ & $\begin{array}{l}\text { Schlum } \\
\text { berger }\end{array}$ & $\begin{array}{l}\text { Casing- } \\
\text { cement } \\
\text { interface }\end{array}$ & $20 \mathrm{kHz}$ & Poor - .91m & $\begin{array}{l}\text { Mostly for } \\
\text { evaluation of } \\
\text { cement-casing } \\
\text { interface/ } 260 \text { C / } 30 \\
\text { ksi }\end{array}$ \\
\hline $\begin{array}{l}\text { USI - Ultrasonic } \\
\text { Imager }\end{array}$ & $\begin{array}{l}\text { Schlum } \\
\text { berger }\end{array}$ & $\begin{array}{l}\text { Casing- } \\
\text { cement } \\
\text { interface }\end{array}$ & $\begin{array}{l}200- \\
700 \mathrm{kHz}\end{array}$ & $\begin{array}{l}30 \mathrm{~mm} \\
\text { lateral }^{2} \\
15.24 \mathrm{~cm} \\
\text { vert }\end{array}$ & 177 C/ 20 ksi \\
\hline $\begin{array}{l}\text { UBI - Ultrasonic } \\
\text { Borehole Imager }\end{array}$ & $\begin{array}{l}\text { Schlum } \\
\text { berger }\end{array}$ & $\begin{array}{l}\text { Full } \\
\text { Borehole, } \\
\text { all } \\
\text { standard } \\
\text { sizes }\end{array}$ & $\begin{array}{l}250,500 \\
\mathrm{kHz}\end{array}$ & "high" & $\begin{array}{l}\text { Not influenced by } \\
\text { mud type, advanced } \\
\text { software }\end{array}$ \\
\hline $\begin{array}{l}\text { OBMI - Oil- } \\
\text { Based } \\
\text { Microlmager }\end{array}$ & $\begin{array}{l}\text { Schlum } \\
\text { berger }\end{array}$ & 3.5 in & $\mathrm{N} / \mathrm{A}$ & $\begin{array}{l}\text { Features as } \\
\text { small as } 1 \mathrm{~cm}\end{array}$ & $\begin{array}{l}\text { Microresistivity } \\
\text { imaging in } \\
\text { nonconductive oil- } \\
\text { based systems }\end{array}$ \\
\hline $\begin{array}{l}\text { ARI - Azimuthal } \\
\text { Resistivity } \\
\text { Imager }\end{array}$ & $\begin{array}{l}\text { Schlum } \\
\text { berger }\end{array}$ & 100 in? & $\mathrm{N} / \mathrm{A}$ & $\begin{array}{l}8 \text { inches } \\
\text { vert } / 60^{\circ} \\
\text { azim angle } \\
\text { for } 1 \text { inch } \\
\text { standoff }\end{array}$ & $\begin{array}{l}177 \mathrm{C} / 20 \mathrm{ksi} / \\
\text { resistivity }\end{array}$ \\
\hline $\begin{array}{l}\text { AIT - Array } \\
\text { Induction } \\
\text { Imager Tools } \\
\text { (multiple) } \\
\end{array}$ & $\begin{array}{l}\text { Schlum } \\
\text { berger }\end{array}$ & $10-90$ in & $\mathrm{N} / \mathrm{A}$ & $\begin{array}{l}.61 \mathrm{~m} \text { or } \\
.30 \mathrm{~m} \text { smooth } \\
\text { borehole }\end{array}$ & $\begin{array}{l}125 \text { C- } 260 \text { C/ } 15 \text { - } \\
30 \text { ksi/ } 3600 \text { feet per } \\
\text { hour log speed/ } \\
\text { resistivity }\end{array}$ \\
\hline $\begin{array}{l}\text { Isolation } \\
\text { Scanner Tool }\end{array}$ & $\begin{array}{l}\text { Schlum } \\
\text { berger }\end{array}$ & $\begin{array}{l}\text { Casing } \\
\text { and } \\
\text { annulus } \\
\text { up to } 3 \text { in }\end{array}$ & $\sim 200 \mathrm{kHz}$ & $1.52 \mathrm{~cm}$ vert & $\begin{array}{l}177 \text { C/ } 20 \text { ksi/ } 563 \\
\text { feet per hour log } \\
\text { speed }\end{array}$ \\
\hline Sonic Scanner & $\begin{array}{l}\text { Schlum } \\
\text { berger }\end{array}$ & formation & wide & $<1.82 \mathrm{~m}$ & 177 C/ 20 ksi \\
\hline
\end{tabular}

All reports should be written for public disclosure. Reports should not contain any proprietary or classified information, other information not subject to release, or any information subject to export control classification. If a report contains such information, notify DOE within the report itself. 


\begin{tabular}{|c|c|c|c|c|c|}
\hline XMAC F1 & $\begin{array}{l}\text { Baker } \\
\text { Hughes }\end{array}$ & 100 feet & $\begin{array}{l}\text { Low } \\
\text { frequenc } \\
\text { y dipole } \\
\text { source }\end{array}$ & low & $\begin{array}{l}232 \text { C/ } 30 \text { ksi/ } 50 \\
\text { feet per minute log } \\
\text { speed/ } 4.5 \text { in } \\
\text { minimum borehole } \\
\text { size/ features don't } \\
\text { need to intercept } \\
\text { borehole }\end{array}$ \\
\hline $\begin{array}{l}\text { Array Dielectric } \\
\text { eXplorer } \\
\text { formation } \\
\text { evaluation } \\
\text { service }\end{array}$ & $\begin{array}{l}\text { Baker } \\
\text { Hughes }\end{array}$ & 6 in & $\begin{array}{l}\text { N/A } \\
\text { ("multipl } \\
\mathrm{e}^{\prime \prime} \text { ) }\end{array}$ & 3 in & $\begin{array}{l}150 \mathrm{C} / 20 \mathrm{ksi} / \\
\text { Mostly for } \\
\text { determining } \\
\text { mobility of oils and } \\
\text { porosity values as } \\
\text { well as hydrocarbon } \\
\text { saturation/ Acquires } \\
\text { permittivity and } \\
\text { conductivity data/ } \\
5.875 \text { inch min } \\
\text { borehole diameter }\end{array}$ \\
\hline $\begin{array}{l}\text { High-Resolution } \\
\text { Vertilog }\end{array}$ & $\begin{array}{l}\text { Baker } \\
\text { Hughes }\end{array}$ & $\begin{array}{l}5.3 \text { in to } \\
9.425 \text { in } \\
\text { dependin } \\
\text { g on hole } \\
\text { diam } \\
\text { (casing } \\
\text { tool) }\end{array}$ & $N / A$ & ? & $\begin{array}{l}175 \mathrm{C} / 15 \mathrm{ksi} / \text { Uses } \\
\text { Hall effect sensors } \\
\text { for magnetic flux } \\
\text { leakage detection/ } \\
\text { has minimum } \\
\text { operating distance } \\
\text { from tool }\end{array}$ \\
\hline $\begin{array}{l}\text { Digital Magnelog } \\
\text { (DMAG) }\end{array}$ & $\begin{array}{l}\text { Baker } \\
\text { Hughes }\end{array}$ & Casing & multi & ? & $\begin{array}{l}\text { Designed to detect } \\
\text { wall thickness } \\
\text { changes by } \\
\text { measuring magnetic } \\
\text { field shift }\end{array}$ \\
\hline $\begin{array}{l}\text { Acoustic Cement } \\
\text { Bond Log }\end{array}$ & $\begin{array}{l}\text { Baker } \\
\text { Hughes }\end{array}$ & $\begin{array}{l}\text { Casing- } \\
\text { Cement } \\
\text { Interface }\end{array}$ & ? & ? & $\begin{array}{l}177 \mathrm{C} / 17 \mathrm{ksi} / 2.38 \\
\text { in min casing }\end{array}$ \\
\hline $\begin{array}{l}\text { Imaging Caliper } \\
\text { Log }\end{array}$ & $\begin{array}{l}\text { Baker } \\
\text { Hughes }\end{array}$ & Casing & $\begin{array}{l}\text { N/A } \\
\text { (mechani } \\
\text { cal) }\end{array}$ & $\begin{array}{l}.003 \text { in azim } \\
.14 \text { in } \\
\text { vertical at } 11 \\
\frac{11}{16} \text { in casing }\end{array}$ & $\begin{array}{l}150 \mathrm{C} / 15 \mathrm{ksi} / \\
\text { resolution strongly } \\
\text { dependent on } \\
\text { borehole size. Listed } \\
\text { resolution is best, } \\
\text { worst is .013 in } \\
\text { azim, . } 28 \text { in vertical }\end{array}$ \\
\hline
\end{tabular}

All reports should be written for public disclosure. Reports should not contain any proprietary or classified information, other information not subject to release, or any information subject to export control classification. If a report contains such information, notify DOE within the report itself. 


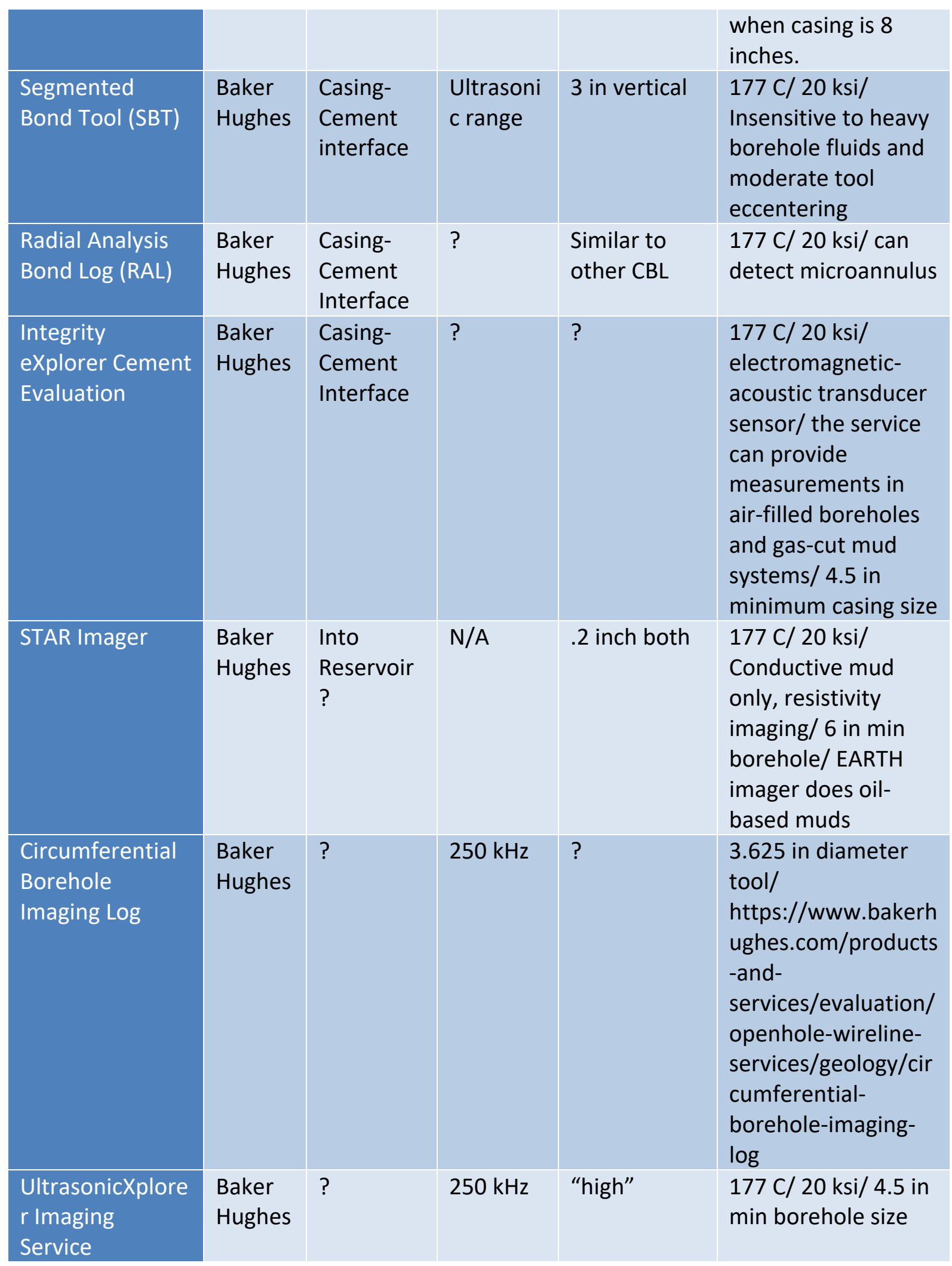

All reports should be written for public disclosure. Reports should not contain any proprietary or classified information, other information not subject to release, or any information subject to export control classification. If a report contains such information, notify DOE within the report itself. 


\begin{tabular}{|c|c|c|c|c|c|}
\hline $\begin{array}{l}\text { GeoXplorer } \\
\text { Imaging Service }\end{array}$ & $\begin{array}{l}\text { Baker } \\
\text { Hughes }\end{array}$ & $\begin{array}{l}\text { Near- } \\
\text { wellbore }\end{array}$ & $\mathrm{N} / \mathrm{A}$ & $\begin{array}{l}.8 \text { in vert } \\
.3 \text { in azim }\end{array}$ & $\begin{array}{l}177 \mathrm{C} / 25 \mathrm{ksi} / \\
\text { Nonconductive mud } \\
\text { systems/ } 7 \text { in min } \\
\text { borehole/ } 79 \% \text { in an } \\
\text { 8-in. (203-mm) } \\
\text { borehole coverage }\end{array}$ \\
\hline $\begin{array}{l}\text { Wellbore } \\
\text { Geometry } \\
\text { Instrument } \\
\text { (WGI) } \\
\text { Measurement } \\
\text { Service }\end{array}$ & $\begin{array}{l}\text { Baker } \\
\text { Hughes }\end{array}$ & $\begin{array}{l}\text { Casing } \\
\text { only }\end{array}$ & $\mathrm{N} / \mathrm{A}$ & 1 in vert & \\
\hline MR eXplorer & $\begin{array}{l}\text { Baker } \\
\text { Hughes }\end{array}$ & 2.1-3.8 in & $\begin{array}{l}\sim 500- \\
1000 \mathrm{kHz} \\
\text { (magneti } \\
\mathrm{c} \\
\text { resonanc } \\
\text { e) }\end{array}$ & high & $\begin{array}{l}175 \mathrm{C} / 20 \mathrm{ksi} / 5.875 \\
\text { in min borehole } \\
\text { size/ Targeted } \\
\text { towards } \\
\text { permeability and } \\
\text { porosity } \\
\text { measurements and } \\
\text { characterize oil }\end{array}$ \\
\hline $\begin{array}{l}\text { Circumferential } \\
\text { Acoustic } \\
\text { Scanning Tool } \\
\text { (CAST) }\end{array}$ & $\begin{array}{l}\text { Hallibu } \\
\text { rton }\end{array}$ & $\begin{array}{l}\text { Casing } \\
\text { and } \\
\text { Cement }\end{array}$ & ? & $\begin{array}{l}1.8^{\circ} \text { azim } \\
\text { openhole } \\
3.6^{\circ} \\
\text { casedhole, } .3 \\
\text { in vert }\end{array}$ & $\begin{array}{l}175 \mathrm{C} / 20 \mathrm{ksi} / 5 \text { in } \\
\text { borehole min/ } \\
\text { DeepSuite CAST-XR } \\
35 \mathrm{ksi}\end{array}$ \\
\hline $\begin{array}{l}\text { Multifinger } \\
\text { Imaging Tool } \\
\text { (MIT) }\end{array}$ & $\begin{array}{l}\text { Hallibu } \\
\text { rton }\end{array}$ & Casing & N/A & ? & $\begin{array}{l}177 \mathrm{C} / 20 \mathrm{ksi} / \\
\text { resolution similar to } \\
\text { competitors, } \\
\text { completely } \\
\text { mechanical }\end{array}$ \\
\hline $\begin{array}{l}\text { Acoustic } \\
\text { Conformance } \\
\text { Xaminer }^{\circledR} \\
\left(\mathrm{ACX}^{\mathrm{TM}}\right)\end{array}$ & $\begin{array}{l}\text { Hallibu } \\
\text { rton }\end{array}$ & $\begin{array}{l}\text { Casing } \\
\text { and } \\
\text { Cement? }\end{array}$ & $?$ & high & $\begin{array}{l}\text { Used to triangulate } \\
\text { leak sources }\end{array}$ \\
\hline $\begin{array}{l}\text { Electromagnetic } \\
\text { Pipe Xaminer }{ }^{\circledR} \mathrm{V} \\
\left(\text { EPX }^{\mathrm{TM}} \mathrm{V}\right) \text { Tool }^{-}\end{array}$ & $\begin{array}{l}\text { Hallibu } \\
\text { rton }\end{array}$ & $\begin{array}{l}\text { Casing } \\
\text { only }\end{array}$ & N/A & $\begin{array}{l}.015 \text { inch } \\
\text { accuracy } \\
\text { defect } \\
\text { detection }\end{array}$ & $\begin{array}{l}177 \mathrm{C} / 15 \mathrm{ksi} / \text { Used } \\
\text { exclusively to } \\
\text { characterize extent } \\
\text { of damage in casing } \\
\text { after already being } \\
\text { picked up by } \\
\text { another method }\end{array}$ \\
\hline
\end{tabular}

All reports should be written for public disclosure. Reports should not contain any proprietary or classified information, other information not subject to release, or any information subject to export control classification. If a report contains such information, notify DOE within the report itself. 


\begin{tabular}{|c|c|c|c|c|c|}
\hline $\begin{array}{l}\text { Xaminer }^{\circledR} \text { Sonic } \\
\text { Imager Service } \\
\text { (also Full Wave } \\
\text { Sonic Tool } \\
\text { (FWS)) }\end{array}$ & $\begin{array}{l}\text { Hallibu } \\
\text { rton }\end{array}$ & formation & low? & Low? & $\begin{array}{l}177 \text { C/ } 20 \text { ksi/ } \\
\text { Measures formation } \\
\text { p-wave velocity } \\
\text { using farthest- } \\
\text { spaced monopole/ } \\
\text { Mostly measures } \\
\text { slowness }\end{array}$ \\
\hline $\begin{array}{l}\text { Radial Cement } \\
\text { Bond Log } \\
\left(\mathrm{RCBL}^{\mathrm{TM}}\right)\end{array}$ & $\begin{array}{l}\text { Hallibu } \\
\text { rton }\end{array}$ & $\begin{array}{l}\text { Cement- } \\
\text { Formation } \\
\text { Interface }\end{array}$ & ? & ? & $\begin{array}{l}\text { Similar capabilities } \\
\text { to other CBL tools }\end{array}$ \\
\hline $\begin{array}{l}\text { SPACE } \\
\text { Panorama } \\
\end{array}$ & Archer & Casing & $3.5 \mathrm{MHz}$ & $\begin{array}{l}.39 \text { in vert } \\
1.25^{\circ} \text { azim }\end{array}$ & $150 \mathrm{C} / 15 \mathrm{ksi}$ \\
\hline $\begin{array}{l}\text { ABI-40 } \\
\text { Televiewer/ } \\
\text { WellCAD }\end{array}$ & $\begin{array}{l}\text { Advanc } \\
\text { e Logic } \\
\text { Technol } \\
\text { ogy }\end{array}$ & $\begin{array}{l}2 " \text { to } 20^{\prime \prime} \\
\text { diameter } \\
\text { depending } \\
\text { on mud } \\
\text { conditions }\end{array}$ & $\sim 1.2 \mathrm{MHz}$ & $\begin{array}{l}\text { Caliper } \\
\text { Resolution: } \\
0.08 \mathrm{~mm}\end{array}$ & $\begin{array}{l}\text { Logging Speed: } 3 \text { to } 24 \\
\mathrm{ft} / \mathrm{min} \\
\text { Samples/revolution: } \\
\text { up to } 360\end{array}$ \\
\hline
\end{tabular}

\section{Explanation of Variance: N/A}

4. Plans for Next Quarter: Task 1, Investigation of existing technology, is complete. However, if anything new shows up on the market, we will perform the same comparison between our technology and any new one.

Task 2. Define metrics

\section{Planned Activities:}

Identify key performance metrics that will be used to judge success in subsequent phases of the project.

2. Actual Accomplishments:

Identified key performance metrics, defined previously in the proposal.

Year 1

3rd quarter progress measure: Determined resolution for channeling outside casing. 4th quarter progress measure: Performed successful tests on wellbores with foamed cements, with similar resolution as for neat cements.

Phase 2 SMART milestone - Go/No-Go2 (end of year 1): Detect defects at the cementformation interface, with high resolution ( $5 \mathrm{~mm}$, or better). Key technical risk: acoustic signal can get trapped in the metal casing, which can lead to minimal acoustic energy transmission behind the casing. Risk minimization: we are using an acoustic source with unique properties (recently patented, with more patents pending). We proved in the past that our low frequency collimated acoustic beam, with the right choice of frequencies (avoiding tube modes), can penetrate successfully through metal casing, and we are planning to further improve the source, and also the receivers, for better signal-to-noise

All reports should be written for public disclosure. Reports should not contain any proprietary or classified information, other information not subject to release, or any information subject to export control classification. If a report contains such information, notify DOE within the report itself. 
ratio. Success metric: defects detection at the cement-formation interface with a resolution of at least $5 \mathrm{~mm}$.

\section{Year 2}

2nd quarter progress measure: Progress toward tool ruggedization for work in adverse conditions.

3rd quarter progress measure: Demonstrated progress toward experimental technique and image processing refinement.

4th quarter progress measure: Improved detection range through foamed cements (these are more attenuating than neat cements).

Phase 3 SMART milestone (end of year 2): Go/No-Go3: Tool survival in adverse conditions of corrosiveness, high temperature and high pressure (brines specific to geothermal wells, at temperatures of at least 250C, and high pressures of $45 \mathrm{kpsi}$ ); Go/NoGo4: Imaging capabilities out in the formation, up to 3 meters. Key technical risk 1: tool cannot survive the adverse conditions of corrosive media, high temperature and high pressures. Risk 1 minimization: in the past, we have developed an acoustic-based sensor that can withstand the aforementioned adverse conditions, and we are planning to leverage on the past knowledge. Key technical risk 2: limited penetration in the formation. Risk 2 minimization: we are planning (tasks 1 and 2), to further enhance the acoustic source and the receivers, such that (1) more power can be transmitted in the formation, and (2) smaller/noisier signal can be detected. Success metric 1: imaging system can survive in adverse conditions of temperature, pressure and corrosiveness. Success metric 2: defects/features (up to $\sim 3 m$ ) can be resolved in the received signal.

Year 3

2nd quarter progress measure: 1st version of the prototype is functional in laboratory tests. Final success metrics:

Prototype in field functionality similar to the one observed in tests in the laboratory.

3. Explanation of Variance: N/A

4. Plans for Next Quarter: This task, Define metrics, is complete. If any additional input is received, either from collaborators, the program side, or competition, this will be taken into account and will be incorporated in our technology.

Task 3. Industry partners/technology maturation plan

1. Planned Activities:

Identify industry partners and use cases for the tools to be further developed in subsequent phases, and provide a technology maturation plan.

2. Actual Accomplishments:

Identified Chevron as potential future partner for further developing the proposed technique. Obtained Support Letter from Chevron, on two different occasions: once when the proposal was submitted, and when we submitted an R\&D100 application.

Technology maturation plan is reflected in the work proposed in the third year:

- Develop an engineering prototype. The prototype has to meet the standards required for $\mathrm{CO} 2$ sequestration and geothermal industry, in terms of resistance

All reports should be written for public disclosure. Reports should not contain any proprietary or classified information, other information not subject to release, or any information subject to export control classification. If a report contains such information, notify DOE within the report itself. 
to corrosion, high temperature and high pressure.

- Develop robust operation \& analysis software, for near real-time 3D imaging by speeding up the measurement and analysis process.

3. Explanation of Variance: N/A

4. Plans for Next Quarter: N/A. Work towards Technology Maturation will be performed in the third year.

\section{PROJECT MANAGEMENT}

Issues, Risks, and Mitigation (If Applicable): N/A

Changes in Approach (If Applicable): N/A

Key Personnel (If Applicable): N/A

Recipient Requests for DOE Attention (If Applicable): N/A

\section{PROJECT OUTPUT}

Publications (If Applicable): N/A

Technologies/Techniques (If Applicable): The main focus of this project is the development of a unique acoustic source that consists of a collimated beam of low frequency, with a bandwidth between 10 and $150 \mathrm{kHz}$. The source will be able to rotate azimuthally for $360 \mathrm{deg}$, move vertically along the borehole, and vary angle of incident beam with respect to borehole axis, such that the environment around the borehole is fully investigated. The acoustic source, incorporated in a logging tool, equipped with appropriate receivers, can provide information in several areas: mapping of the borehole shape and borehole casing, cement bond quality logging, mapping of the area around the borehole, fault detection, fracture detection, porosity mapping, fluid saturation mapping, fluid flow paths, etc.

Status Reports (If Applicable): N/A

Media Reports (If Applicable): N/A

Invention Disclosures (If Applicable): N/A

Patent Applications (If Applicable): submitted patent on low-frequency collimated acoustic source: SIMPLE BESSEL-LIKE COLLIMATED SOUND BEAM GENERATOR

All reports should be written for public disclosure. Reports should not contain any proprietary or classified information, other information not subject to release, or any information subject to export control classification. If a report contains such information, notify DOE within the report itself. 
Licensed Technologies (If Applicable): N/A

Networks/Collaborations Fostered (If Applicable): N/A

Websites Featuring Project Work or Results (If Applicable): N/A

Other Products (If Applicable): N/A

Awards, Prizes, and Recognition (If Applicable): N/A

All reports should be written for public disclosure. Reports should not contain any proprietary or classified information, other information not subject to release, or any information subject to export control classification. If a report contains such information, notify DOE within the report itself. 


\section{REFERENCES}

Davatzes, N.C. and Hickman, S., 2005. Comparison of acoustic and electrical image logs from the Coso geothermal field, CA. In Proceedings, Thirtieth Workshop on Geothermal Reservoir Engineering, Stanford University (pp. 1-11).

Massiot, C., D.D. McNamara, A. Nicol, J. Townend, 2015. Fracture width and spacing distributions from borehole televiewer logs and cores in the Rotokawa Geothermal Field, New Zealand. Proceedings World Geothermal Congress 2015, Melbourne, Australia, 19-25 April 2015.

Prensky, S. 1999. Advances in Borehole Imaging Technology and Applications. In Borehole Imaging: Applications and Case Histories, ed. M. Lovell, G. Williamson, and P. Harvey, 159, 143. London: Geological Soc. Special Publications.

Zemanek, J., Glenn, E.E., Norton, L.J. et al. 1970. Formation evaluation by inspection with the borehole televiewer. Geophysics 35 (2): 254-269. http://dx.doi.org/10.1190/1.1440089.

All reports should be written for public disclosure. Reports should not contain any proprietary or classified information, other information not subject to release, or any information subject to export control classification. If a report contains such information, notify DOE within the report itself. 


\section{APPENDIX A - COMPARISON MATRIX}

\begin{tabular}{|l|c|c|c|c|}
\hline Parameter & ACCObeam & $\begin{array}{c}\text { Standard borehole } \\
\text { sonic probe, e.g. } \\
\text { BARS } \\
\text { SCHLUMBERGER }\end{array}$ & $\begin{array}{c}\text { Ultrasonic probe, } \\
\text { e.g. UBI } \\
\text { SCHLUMBERGER }\end{array}$ & $\begin{array}{c}\text { SPACE } \\
\text { Panorama } \\
\text { ARCHER }\end{array}$ \\
\hline Frequency & $10-250 \mathrm{kHz}$ & $0.3-8 \mathrm{kHz}$ & 250, or $500 \mathrm{kHz}$ & $3.5 \mathrm{MHz}$ \\
\hline
\end{tabular}

Comments: Low frequency is needed for deeper penetration, but conventional low-frequency sources have a very large beam spread, which decreases resolution. Our approach provides a low-frequency beam that is still collimated and has no side lobes.

\begin{tabular}{|l|l|l|l|l|}
\hline $\begin{array}{l}\text { Penetration } \\
\text { Range }\end{array}$ & $3 \mathrm{~m}$ & $15 \mathrm{~m}$ & Casing $(<10 \mathrm{~mm})$ & Casing only \\
\hline
\end{tabular}

Comments: ACCObeam is collimated and has a much longer investigation range than other highfrequency sources, which leads to good understanding of both the borehole and the surrounding environment. Although it does not currently penetrate as far as standard probes, ACCObeam's collimation means that it surpasses standard probes in critical ways, namely imaging resolution (ACCObeam's resolution is 60-100 times better than standard sonic probes) and power efficiency. ACCObeam is also adaptable for numerous other applications.

\begin{tabular}{|l|c|c|c|c|}
\hline Resolution & $3-5 \mathrm{~mm}$ & $300 \mathrm{~mm}$ & $4-5 \mathrm{~mm}$ & $10 \mathrm{~mm}$ \\
\hline
\end{tabular}

Comments: ACCObeam has the deep penetration advantage of a low-frequency source, but it maintains the same resolution as higher-frequency sources. This resolution is critical because even small fractures, delaminations, and other defects can lead to catastrophic failure of a borehole.

\begin{tabular}{|l|l|c|c|c|}
\hline Collimation & $<6 \mathrm{deg}$ & $\begin{array}{c}\text { Monopole/dipole } \\
>180 \mathrm{deg}\end{array}$ & $<15 \mathrm{deg}$ & $<6 \mathrm{deg}$ \\
\hline
\end{tabular}

Comments: Our source has a very similar collimation/beam spread as its high-frequency competitors, even though it generates a low-frequency beam that penetrates much deeper than any high-frequency source. Please note the huge difference when compared with the standard borehole sonic probe.

\begin{tabular}{|l|c|c|c|c|}
\hline Cost & $\$$ & $\$ \$ \$ \$$ & $\$ \$ \$ \$$ & $\$ \$ \$$ \\
\hline
\end{tabular}

Comments: All other products are offered as a service/package operated by a specific company, so the cost of using their source is thousands of dollars. ACCObeam costs $\$ 50-\$ 200$ to manufacture and is compatible with virtually any imaging software or hardware.

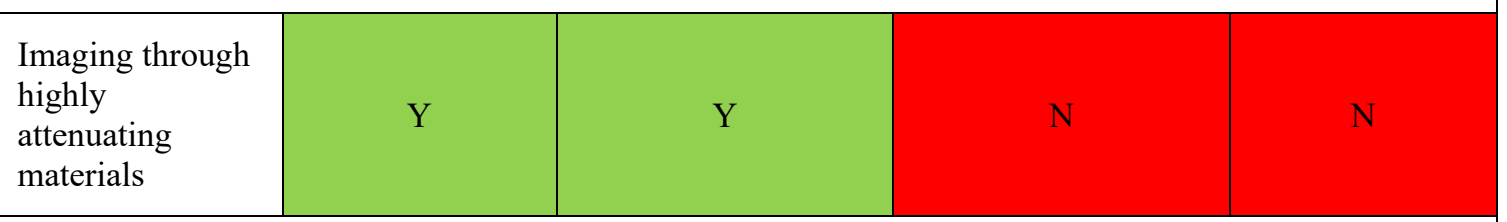

Comments: The only other product able to image clearly and beyond casing in highly attenuating media (such as concrete, drilling mud, rock, and bone) is the low-frequency standard borehole sonic probe.

All reports should be written for public disclosure. Reports should not contain any proprietary or classified information, other information not subject to release, or any information subject to export control classification. If a report contains such information, notify DOE within the report itself. 


\begin{tabular}{|c|c|c|c|c|}
\hline Parameter & Your product & $\begin{array}{c}\text { Standard borehole } \\
\text { sonic probe, e.g. } \\
\text { BARS } \\
\text { Schlumberger }\end{array}$ & $\begin{array}{c}\text { Ultrasonic probe, } \\
\text { e.g. UBI } \\
\text { Schlumberger }\end{array}$ & $\begin{array}{c}\text { SPACE } \\
\text { Panorama } \\
\text { Archer }\end{array}$ \\
\hline Size & Small/compact & Large & Small/compact & Small/compact \\
\hline
\end{tabular}

Comments: ACCObeam is similar in size to high-frequency sources, but much smaller than a typical low-frequency source.

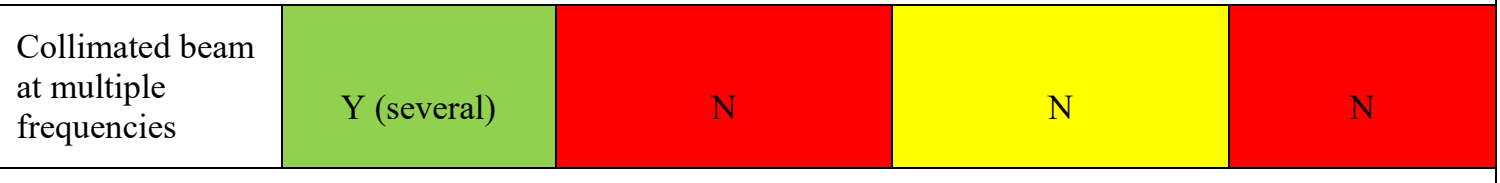

Comments: The UBI ultrasonic probe can provide either a somewhat collimated $250 \mathrm{kHz}$ beam or a more collimated $500 \mathrm{kHz}$ beam, but our source images at numerous frequencies ranging from 10-250 $\mathrm{kHz}$ while maintaining a tight collimation.

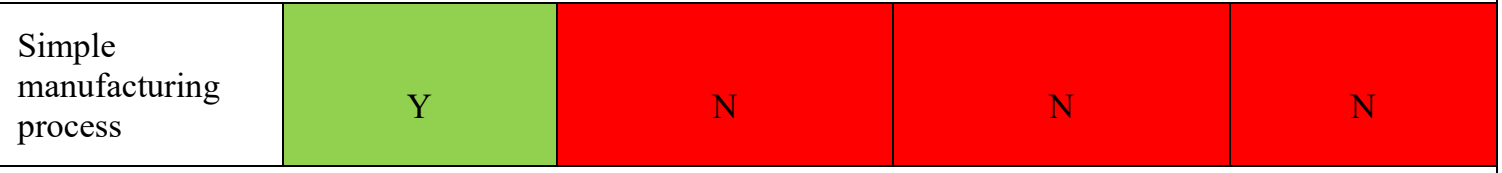

Comments: ACCObeam's design requires radial clamping, achieved by gluing a piezoelectric transducer into casing made of any strong material. Comparative technologies require filler material and a small matching inductor for "loading" the back of the transducer, making the packaging process more sophisticated.

Images through various media Y

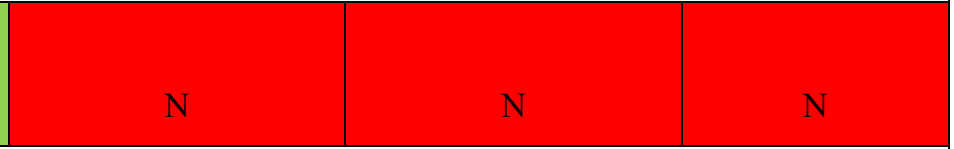

Comments: No competing source can be used to image in assorted media. Since our source operates at a low frequency, it can transmit or receive in liquids, solids, gases, and air.

\begin{tabular}{|l|l|l|l|}
\hline $\begin{array}{l}\text { Frequency } \\
\text { hopping }\end{array}$ & $\mathrm{Y}$ & $\mathrm{N}$ & $\mathrm{Y}$ (limited) \\
\hline
\end{tabular}

Comments: ACCObeam supports as many as 4-10 frequencies, while the UBI has only two selectable frequencies.

All reports should be written for public disclosure. Reports should not contain any proprietary or classified information, other information not subject to release, or any information subject to export control classification. If a report contains such information, notify DOE within the report itself. 